\title{
Renal protective effect of Rosa laevigata Michx. by the inhibition of oxidative stress in streptozotocin-induced diabetic rats
}

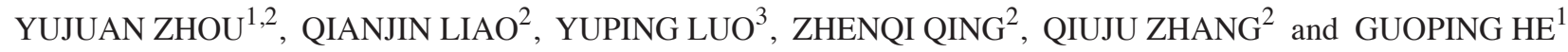 \\ ${ }^{1}$ Central South University, Changsha, Hunan 410083; ${ }^{2}$ University of South China, Hengyang, Hunan 421001; \\ ${ }^{3}$ The Second Hospital of The University of South China, Hengyang, Hunan 421001, P.R. China
}

Received January 6, 2012; Accepted March 29, 2012

DOI: $10.3892 / \mathrm{mmr} .2012 .855$

\begin{abstract}
Diabetic nephropathy (DN) is a long-term complication of diabetic mellitus. Numerous reports have suggested that oxidative stress is defined as the excessive production of reactive oxygen species (ROS) surpassing existing anti-oxidative defense mechanisms, and plays a critical role in the pathogenesis of diabetic nephropathy. Rosa laevigata Michx. (RLM) is the commonly used traditional Chinese medicine for the treatment of chronic urinary tract infections and anti-oxidative treatments, and has been shown to have a renal protective effect in diabetic rats. In the present study, we further investigate the effects of RLM on oxidative stress in the kidneys of streptozotocin-induced diabetic rats with DN. Our results suggest that RLM significantly ameliorates renal dysfunction in diabetic rats. The protection against the development of DN by RLM treatment involves increasing the activity of superoxide dismutase and total anti-oxidant capacity, decreasing the levels of malondialdehyde and ROS, and inhibiting the expression of nuclear factor- $\kappa \mathrm{B}$ p65 and monocyte chemoattractant protein-1 at both the protein and mRNA levels with a concomitant increase in the expression of the I $\kappa \mathrm{B} \alpha$ protein. These results highlight the potential therapeutic application of RLM for the treatment of DN.
\end{abstract}

\section{Introduction}

Diabetic nephropathy (DN) is the most common cause of endstage renal disease (1). The pathogenesis of DN is complex, and multiple pathways and factors that lead to kidney injury are involved, including oxidative stress (OS) (2). There is strong evidence that OS, which precedes the development of endothelial cell dysfunction, plays a key role in the pathogenesis of

Correspondence to: Dr Guoping He, Central South University, 172 Tong-Zhipo Road, Changsha, Hunan 410083, P.R. China E-mail: hgpcsu@yahoo.com.cn

Dr Qiuju Zhang, University of South China, 28 Chang-Sheng West Road, Hengyang 421001, Hunan, P.R. China

E-mail: jqz0907@163.com

Key words: Rosa laevigata Michx., diabetic nephropathy, oxidative stress, nuclear factor- $\kappa \mathrm{B}$, monocyte chemoattractant protein-1
DN (2-4). OS is the imbalance of oxidants and anti-oxidants in favour of oxidants, which lead to the disruption of redox signaling and/or molecular damage (5). OS is estimated by the production of pro-oxidants, such as superoxide, by the expression or activity of an anti-oxidant, such as superoxide dismutase (SOD), or by the damage induced by the OS of biomolecules, such as proteins (nitration of tyrosine residues, nitrotyrosine) and lipids (aldehydes from lipid peroxidation). Reactive oxygen species (ROS) play an important role in OS, and much evidence suggests that diabetes triggers multiple pathways for the increased generation of ROS (6). These include enhanced glucose oxidation, increased mitochondrial superoxide production and the production of advanced glycation end-products $(7,8)$. SOD, the first-line endogenous defence against superoxide production, converts superoxide $\left(\mathrm{O}_{2}^{-}\right)$to hydrogen peroxide $\left(\mathrm{H}_{2} \mathrm{O}_{2}\right)$, which is degraded to water and molecular oxygen by catalase and glutathione peroxidase (9). Reduction in OS ameliorates renal dysfunction in DN rats (10). Therefore, the improvement of the anti-oxidant system and the reduction in OS may be a potential therapeutic target for DN.

Herbal medicines are naturally occurring, and herbal medicines or their extracts have been used to cure various diseases (11). The active herbal compounds may be flavonoids, saponins and lipids, and are extensively present in plants, fruits and vegetables (12). Rosa laevigata Michx. (RLM) (Rosaceae) is an evergreen climbing shrub widely distributed throughout southern China (13). The leaves of RLM are widely used as a traditional Chinese folk medicine for the treatment of skin tumours, burns and ulcers (14). These fruits, which are known as the commonly used traditional Chinese medicine, Jin Ying $\mathrm{Zi}$, are listed in the Chinese Pharmacopoeia for the treatment of chronic urinary tract infections, wet dreams, urinary incontinence, uterine prolapses, menstrual irregularities and leucorrhea $(13,14)$. RLM inhibits the growth of the influenza virus and Bacillus dysenteriae in vitro, and it has long been used for anti-inflammatory treatments. In addition, it is also used for anti-oxidative treatments (15). However, the molecular mechanisms supporting its clinical claims remain elusive.

Our previous studies have shown that RLM treatment decreases the blood glucose and lipid levels in rabbits fed a high-glucose and -lipid diet (16), and protects the kidneys of streptozotocin (STZ)-induced diabetic rats via decreasing the expression of TGF- $\beta 1$, collagen type IV and monocyte chemoattractant protein-1 (MCP-1) $(17,18)$. Thus, in the present study 
we further investigated the renal protective effect of RLM by the inhibition of OS in STZ-induced diabetic rats, and assessed its usefulness as a potential agent for the treatment of DN.

\section{Materials and methods}

Preparation of botanical extracts. The aqueous extracts of RLM fruits $(2.5 \mathrm{~g} / \mathrm{ml})$ were purchased from an established local company, Pharmaceutical Ltd. (Hengyang, China). Briefly, RLM crude fruits were ripped and the seeds were abandoned, then soaked in water for $30 \mathrm{~min}$ and added in the Chinese herbal medicine tisanes machine (ZG-280 Zhong Cheng Pharmaceutical Machinery Factory Hunan) to obtain the aqueous extracts of RLM fruits.

Animals and experimental protocols. Sixty male SpragueDawley (SD) rats, weighing $250 \pm 10 \mathrm{~g}$, were obtained from the Centre of Experimental Animals at the University of South China in Hunan, China [animal quality certificate SYXK (Hunan Province) 2009-0011]. The temperature and humidity in the animal room were carefully controlled at $25^{\circ} \mathrm{C}$ and $70 \%$, respectively. All experiments were carried out in accordance with the China Animal Welfare Legislation and were approved by the University of South China Committee on Ethics in the Care and Use of Laboratory Animals.

In order to created the diabetic model, $40 \mathrm{SD}$ rats, which were previously fed a high-fat/high-sucrose/high-cholesterol diet for 2 months, were administered STZ (Sigma, St. Louis, MO, USA; Lot 2126F) in a single dose of $65 \mathrm{mg} / \mathrm{kg}$ intraperitoneally (i.p.) in citrate buffer as previously described (19). Fasting blood glucose (FBG) levels were measured on the 3rd day after STZ injection using the SinoCare SXT blood glucose meter (SinoCare Corp., Changsha, China). The animals were considered diabetic if FBG levels were $>14$ mmol/l (20). Thirty diabetic rats were randomly selected and evenly divided into 2 groups comprising 15 animals in each group as follows: diabetic model group (DM, $n=15)$ and diabetic + RLM group (DM + RLM, n=15, fed with aqueous extracts of RLM, $5 \mathrm{~g}$ / $\mathrm{kg}$ /day) (18). Another 20 normal SD rats were divided into 2 groups comprising 10 animals in each group as follows: normal control group (control, $\mathrm{n}=10$ ) and normal control + RLM group (control + RLM, $n=10$, fed with aqueous extracts of $\mathrm{RLM}, 5 \mathrm{~g} / \mathrm{kg} / \mathrm{day})$. The aqueous extracts of RLM were injected daily into the stomach.

All the rats were allowed access to water ad libitum, and weighed before the experiment and 1 day prior to sacrifice. Urine was collected from the rats housed in metabolic cages for $24 \mathrm{~h}$. All animals were sacrificed at the end of the 24 th week. Blood was collected from a puncture of the inferior vena cava at the time of sacrifice. The serum was separated by centrifugation and was stored at $-20^{\circ} \mathrm{C}$ until the analysis. Kidney samples were rapidly excised, decapsulated, weighed and frozen in liquid nitrogen or fixed in $10 \%$ buffered formaldehyde solution or glutaraldehyde (3\% in PBS).

Histopathological examination of kidneys. For routine histopathological examination by light microscopy, a coronal slice of the kidney was processed in the standard fashion, and the sections were stained with H\&E and periodic acid-Schiff (PAS). The average area and volume of the glomerulus were measured using the HPIAS-2000 Image Analysis System V6.0. Mean values were calculated for each of the 10 glomeruli per section. Glutaraldehyde (3\% in PBS)-fixed renal tissues were post-fixed with $1 \% \mathrm{OsO}_{4}$ for $90 \mathrm{~min}$ and dehydrated with graded ethanol. Samples were embedded with resin (Epon 812) and polymerised at $72^{\circ} \mathrm{C}$ for $48 \mathrm{~h}$. Ultrasections $(50-70 \mathrm{~nm})$ were stained with lead acetate and uranyl and were observed using a JEM1230 microscope (Tokyo, Japan). The images were captured with a Gatan imaging system (Gatan Inc., NY, USA).

Biochemical analysis of blood. Serum samples were collected for the measurement of total cholesterol (TC), triglyceride (TG), blood urea nitrogen (BUN) and serum creatinine (Scr) levels. Every $24 \mathrm{~h}$, the urine from each rat was collected from the metabolic cage to measure urinary albumin. Each of these measurements was performed according to the manufacturer's instructions (Nanjing Jiancheng Biomedical Engineering Co. Ltd., Nanjing, China).

Anti-oxidant defenses and OS biomarkers in kidney homogenates. Kidney homogenates $(10 \%, \mathrm{w} / \mathrm{v})$ were prepared with $0.1 \mathrm{M}$ PBS and centrifuged at $12,000 \mathrm{x} \mathrm{g}$ for $10 \mathrm{~min}$. The supernatant was used to determine the total anti-oxidant capacity (T-AOC), SOD and malondialdehyde (MDA) levels with commercially available kits (Nanjing Jiancheng Biomedical Engineering Co. Ltd.) and ROS levels with a commercially available kit (Shanghai Yueyan Biological Technology Co., Ltd., Shanghai, China). The experiments were carried out according to the manufacturer's instructions.

Quantitative real-time PCR ( $q R T$ - $P C R)$. Quantitative realtime PCR analysis was performed as described previously (21). Briefly, total RNA was extracted using the TRIzol extraction kit (Invitrogen, Carlsbad, CA, USA) according to the manufacturer's instructions and then reverse-transcribed by the AMV reverse transcriptase (Promega, Madison, WI, USA). The qRT-PCR assays were performed with a SYBR-Greencontaining PCR kit (GenePharma, Shanghai, China). The primers used to detect nuclear factor $-\kappa \mathrm{B}(\mathrm{NF}-\kappa \mathrm{B})$ and MCP-1 mRNA by qRT-PCR were as follows: NF- $\mathrm{B}$ p 65 forward, 5'-TTCCTCAGCCATGGTACCTC-3' and reverse, 5'-CCCC AAGTCTTCATCAGCAT-3' (GenBank accession no. NM-009045.4); and MCP-1 forward, 5'-CAGAAACCAG CCAACTCTCA-3' and reverse, 5'-AGACAGCACGTGGA TGCTAC-3' (GenBank accession no. AF058786). The mRNA expression was normalised to that of GAPDH. The primer sequences for GAPDH were: forward, 5'-TCAAGAAGGTGG TGAAGCAG-3' and reverse, 5'-AGGTGGAAGAATGGGA GTTG-3' (GenBank accession no. NM-017008). All real-time PCR analyses were performed using the Bio-Rad IQTM5 multicolor real-time PCR detection system (Bio-Rad, Hercules, CA, USA). The relative change in MCP-1 mRNA expression was determined by a fold-change analysis in which the degree of change $=2^{-\Delta \Delta \mathrm{CT}}(22)$.

Western blotting. Total and nuclear proteins were isolated from the kidney tissue of rats using a nuclear and cytoplasmic extraction kit (Pierce Biotechnology, Rockford, IL, USA). Western blot analysis was performed as described previously (23). Briefly, total and nuclear proteins were extracted 
Table I. Effects of RLM on body weight (BW), kidney weight (KW), KW/BW ratio, blood glucose, serum lipid profiles, urine volume and albumin after $24 \mathrm{~h}$ of treatment.

\begin{tabular}{lcccc}
\hline Characteristics & Control & Control + RLM & DM & DM + RLM \\
\hline Body weight $(\mathrm{g})$ & $369.5 \pm 9.60$ & $370.50 \pm 7.80^{\mathrm{c}}$ & $271.2 \pm 14.7^{\mathrm{a}}$ & $347.2 \pm 18.4^{\mathrm{a}, \mathrm{b}}$ \\
Kidney weight $(\mathrm{g})$ & $1.285 \pm 0.10$ & $1.278 \pm 0.17^{\mathrm{c}}$ & $1.503 \pm 0.18^{\mathrm{a}}$ & $1.311 \pm 0.24^{\mathrm{a}, \mathrm{b}}$ \\
KW/BW (mg/g) & $3.40 \pm 0.23$ & $3.50 \pm 0.12^{\mathrm{c}}$ & $5.50 \pm 0.82^{\mathrm{a}}$ & $3.80 \pm 0.94^{\mathrm{b}}$ \\
Fasting blood glucose (mmol/l) & $6.39 \pm 0.97$ & $6.69 \pm 0.76^{\mathrm{c}}$ & $31.37 \pm 2.38^{\mathrm{a}}$ & $19.64 \pm 1.42^{\mathrm{a}, \mathrm{b}}$ \\
Total cholesterol (mmol/l) & $2.54 \pm 0.36$ & $2.64 \pm 0.27^{\mathrm{c}}$ & $4.27 \pm 0.81^{\mathrm{a}}$ & $3.04 \pm 0.38^{\mathrm{b}}$ \\
Triglyceride (mmol/l) & $0.81 \pm 0.57$ & $0.97 \pm 0.48^{\mathrm{c}}$ & $1.82 \pm 0.24^{\mathrm{a}}$ & $1.27 \pm 0.21^{\mathrm{b}}$ \\
Blood urea nitrogen (mmol/l) & $7.55 \pm 0.71$ & $8.13 \pm 0.62^{\mathrm{c}}$ & $17.49 \pm 2.71^{\mathrm{a}}$ & $11.29 \pm 1.75^{\mathrm{a}, \mathrm{b}}$ \\
Serum creatinine (mmol/l) & $70.78 \pm 11.54$ & $71.32 \pm 10.08^{\mathrm{c}}$ & $158.71 \pm 14.83^{\mathrm{a}}$ & $107.34 \pm 15.34^{\mathrm{a}, \mathrm{b}}$ \\
Urine amount (ml/day) & $27.80 \pm 8.38$ & $28.40 \pm 7.36^{\mathrm{c}}$ & $152.50 \pm 25.38^{\mathrm{a}}$ & $108.30 \pm 18.29^{\mathrm{a}, \mathrm{b}}$ \\
Urinary albumin excretion $(\mathrm{mg} / \mathrm{l})$ & $17.30 \pm 4.17$ & $17.50 \pm 4.03^{\mathrm{c}}$ & $89.50 \pm 7.82^{\mathrm{a}}$ & $37.80 \pm 3.16^{\mathrm{a}, \mathrm{b}}$ \\
\hline
\end{tabular}

RLM, Rosa laevigata Michx.; DM, diabetic model group. All data are presented as the means $\pm \mathrm{SD}$. ${ }^{\mathrm{P}} \mathrm{P}<0.05$ vs. the normal group; ${ }^{\mathrm{b}} \mathrm{P}<0.05 \mathrm{vs}$. the model group; ${ }^{\mathrm{C}}>0.05$ vs. the normal group.

following the homogenisation of the renal cortex using a nuclear and cytoplasmic extraction kit (Pierce Biotechnology). The concentration of protein was determined using a $\mathrm{BCA}^{\mathrm{TM}}$ protein assay kit (Pierce Biotechnology) according to the manufacturer's instructions. Protein extracts $(30 \mu \mathrm{g})$ were combined with an equal volume of $2 \mathrm{X}$ loading buffer, boiled for $5 \mathrm{~min}$ and size-fractionated by electrophoresis on $10 \%$ SDS-polyacrylamide gels. Proteins were transferred onto nitrocellulose membranes by electroblotting. Membranes were incubated with 5\% skim milk powder in PBS for $1 \mathrm{~h}$ and were subsequently incubated with primary antibodies for anti-

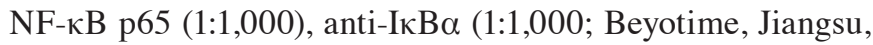
China), MCP-1 (1:1,000; Cell Signaling Technology, USA) and mouse anti- $\beta$-actin $\left(1: 2,000\right.$; Sigma) overnight at $4^{\circ} \mathrm{C}$. After incubation, the samples were washed, incubated with peroxidase-conjugated secondary antibody, detected using enhanced chemiluminescence (Cell Signaling Technology) and captured on X-ray film. Protein levels were quantified densitometrically with a documentation system.

Statistical analysis. Data from these experiments were assessed using SPSS 11.5 software. Results are represented as the means \pm standard deviation (SD). The one-way analysis of variance (ANOVA) and t-test were used for a statistical comparison within and between groups. $\mathrm{P}<0.05$ denoted a statistically significant difference.

\section{Results}

Characteristics of the experimental rats. Table I presents the details of the rat characteristics in the 4 groups at the end of the experimental period. The ratio of kidney weight to body weight $(\mathrm{KW} / \mathrm{BW})$ and average kidney weight $(\mathrm{KW})$ significantly increased, while the body weight (BW) decreased in the diabetic group compared to the normal control group $(\mathrm{P}<0.05)$. RLM treatment maintained $\mathrm{BW}$ and decreased $\mathrm{KW} / \mathrm{BW}$ compared to the DM group $(\mathrm{P}<0.05)$. Blood glucose levels of the diabetic rats were markedly elevated compared to the normal control rats throughout the entire experiment, but the blood glucose levels of the DM + RLM group rats showed a partial improvement at the end of the experiment. TC, TG, BUN and Scr levels were also significantly improved in the DM group compared to the control group, and these heightened levels were partially rescued by RLM treatment. Compared to the control rats, the 24-h urine volume of the DM rats was significantly larger and was partially reduced in the DM + RLM group. There was no significant difference in any of these parameters between the control + RLM and the normal control groups $(\mathrm{P}>0.05)$.

Changes in urinary albumin levels. In order to assess the effects of RLM treatment on the kidney functional abnormalities in DM rats, urinary albumin excretion was measured. The values in the DM rats were markedly higher, while significantly reduced in the DM + RLM group (Table I). There were no significant differences in urinary albumin excretion of rats in the control + RLM group compared to the normal control group. This shows that RLM moderates the kidney functional abnormalities of diabetic rats.

Effect of RLM on T-AOC and SOD activity, MDA and ROS levels in kidney homogenates. Compared to the control group, the T-AOC and SOD levels were significantly reduced in the kidneys of DM rats; instead, the MDA and ROS levels were obviously increased. RLM treatment significantly increased T-AOC and SOD levels and decreased the levels of MDA and ROS in the diabetic rats compared to the diabetic control rats $(\mathrm{P}<0.05)$. However, there were no significant differences in the above parameters between the control + RLM and normal control groups ( $\mathrm{P}>0.05)$ (Fig. 1).

Changes in kidney morphology. We used glomerular volume to evaluate glomerular hypertrophy. The mean glomerular volume of the diabetic rats was $2.58 \times 10^{6} \mu \mathrm{m}^{3}$, which was significantly greater than that of the normal control rats $\left(1.79 \times 10^{6} \mu \mathrm{m}^{3}\right)$. However, the mean glomerular volume in 
A

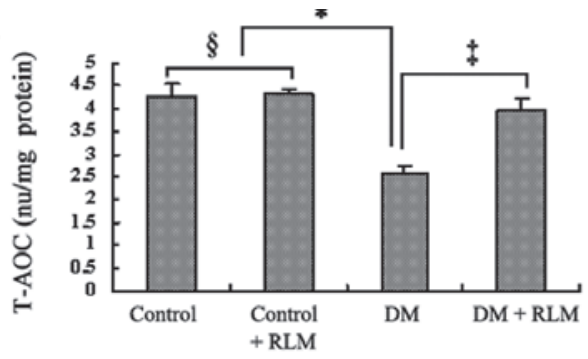

C

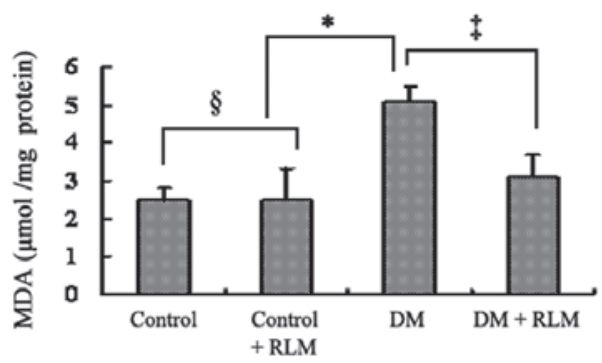

B

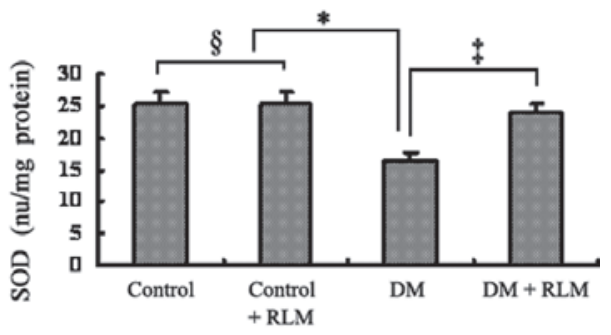

D

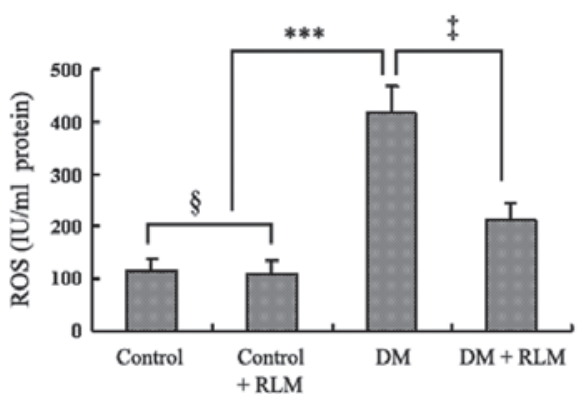

Figure 1. Effects of Rosa laevigata Michx. (RLM) treatment on renal SOD activity and MDA content on diabetic nephropathy in rats. Levels of (A) T-AOC, (B) SOD, (C) MDA and (D) ROS in kidney tissue homogenates from the different groups. ${ }^{*} \mathrm{P}<0.05,{ }^{* * *} \mathrm{P}<0.001$ vs. the normal group; ${ }^{{ }^{\circ}} \mathrm{P}<0.05$ vs. the diabetic model (DM) group; ${ }^{\circledR} \mathrm{P}>0.05$ vs. the normal group. T-AOC, total anti-oxidant capacity; SOD, superoxide dismutase; MDA, malondialdehyde; ROS, reactive oxygen species.

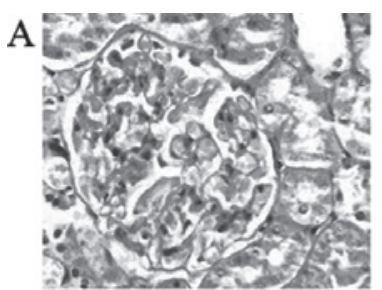

Control

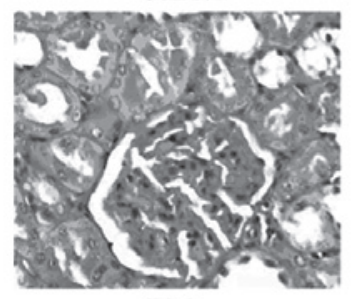

DM

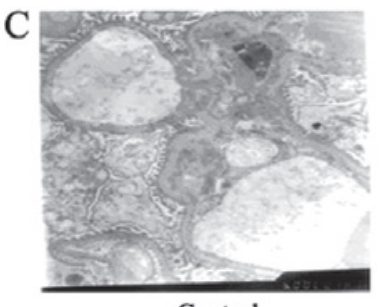

Control

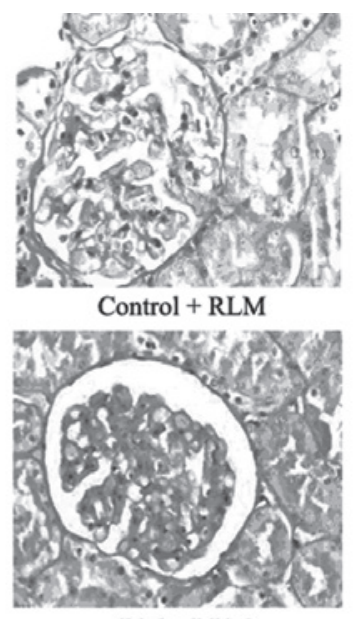

$\mathrm{DM}+\mathrm{RLM}$

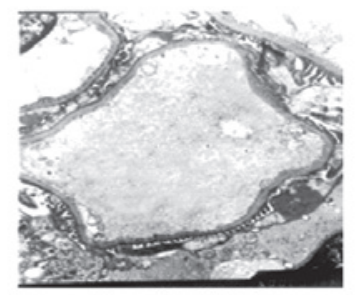

Control + RLM

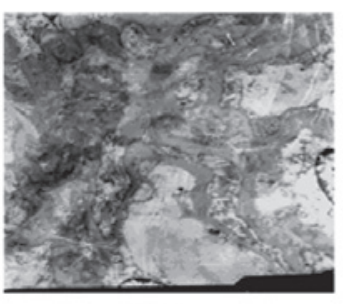

$\mathrm{DM}$

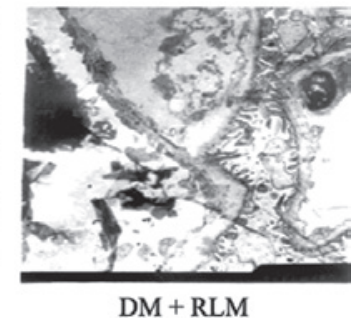

$\mathrm{DM}+\mathrm{RLM}$

Figure 2. Effect of Rosa laevigata Michx. (RLM) treatment on kidney morphology in rats with diabetic nephropathy. (A) Representative photomicrographs of a periodic acid-Schiff (PAS)-stained kidney (original magnification, x400). (B) Quantitative assessment of the mesangial matrix area. (C) The glomerular ultrastructural changes and the thickness of basement membranes were evaluated by electron microscopy (original magnification, $\mathrm{x} 6,000$ ). Data are presented as the means $\pm \mathrm{SD}$. ${ }^{*} \mathrm{P}<0.05$ vs. the normal group; ${ }^{ } \mathrm{P}<0.05$ vs. the diabetic model (DM) group; ${ }^{\circledR} \mathrm{P}>0.05$ vs. the normal group.

the DM + RLM group $\left(2.1 \times 10^{6} \mu \mathrm{m}^{3}\right)$ was less than that of the diabetic rats. There were no significant differences in the glomerular volume of rats in the control + RLM group $\left(1.81 \times 10^{6} \mu \mathrm{m}^{3}\right)$ compared to the normal control group.
Fig. 2A shows the findings of representative histopathology from PAS-stained kidneys of the 4 groups. The mesangial matrix was damaged more extensively in the glomeruli of diabetic rats than in the control rats, and the damage was 
A

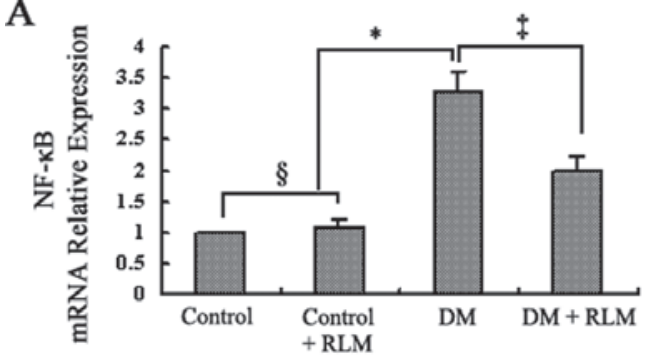

B

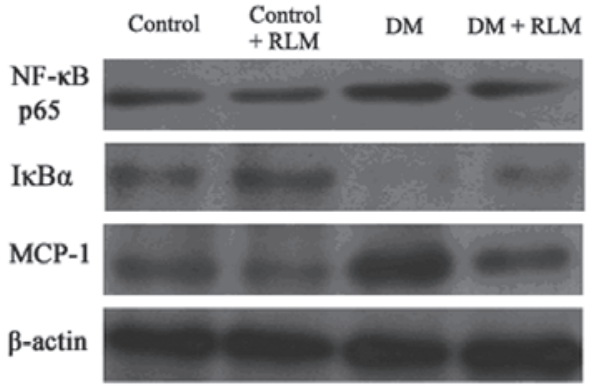

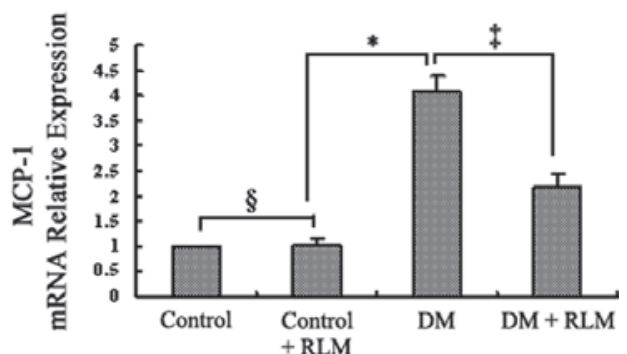

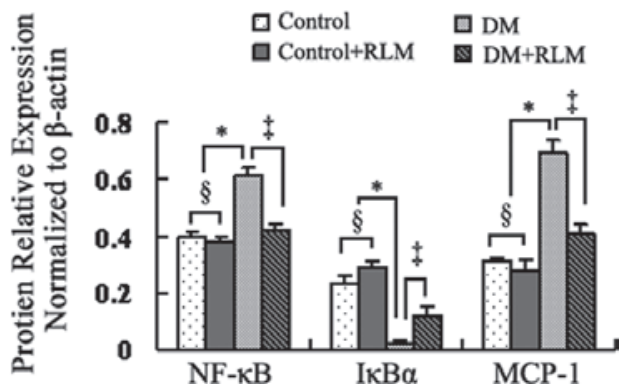

Figure 3. Effect of Rosa laevigata Michx. (RLM) treatment on the expression of NF- $\kappa \mathrm{B}$ p65 and MCP-1 in diabetic rat kidneys. The expression of NF- $\kappa \mathrm{B}$ p65 and MCP-1 in the renal tissues was determined by qRT-PCR and western blotting. (A) mRNA expression levels determined by qRT-PCR. (B) Protein expression levels include I $\kappa \mathrm{B} \alpha$ determined by western blotting. mRNA expression values were normalised to those of GAPDH, and the detection of $\beta$-actin served as the control for equal protein loading. Data present the means \pm SD of 3 independent experiments or of 1 of 3 independent experiments. ${ }^{*} \mathrm{P}<0.05$ vs. the normal group; ${ }^{\ddagger} \mathrm{P}<0.05$ vs. the diabetic model (DM) group; ${ }^{\S} \mathrm{P}>0.05$ vs. the normal group. NF- $\mathrm{B}$, nuclear factor- $\kappa \mathrm{B} ; \mathrm{MCP}-1$, monocyte chemoattractant protein-1.

reduced in the DM + RLM group. Fig. 2B shows the quantitative analysis results of mesangial matrix expansion in all groups. The ratio of the mesangial matrix to the glomerular area in the diabetic rats was markedly higher compared to the normal control rats, while this expansion was significantly reduced in the DM + RLM group.

As a characteristic of early DN is the thickening of the glomerular basement membrane (GBM), we evaluated the ultrastructural glomerular changes and the thickness of the basement membranes by electron microscopy. As shown in Fig. 2C, the GBM thickening and podocyte foot process effacement were striking in the diabetic rats, but these conditions began to revert back to normal following RLM treatment. There were no significant differences in kidney morphology between the normal control and control + RLM groups, which therefore suggested that RLM treatment did not lead to renal injury in the rats.

Effect of RLM on NF- $\mathrm{BB}$ p65 and MCP-1 expression in kidneys. The effect of RLM treatment on NF- $\kappa$ B p 65 and MCP-1 expression in diabetic kidneys was evaluated by qRT-PCR and western blotting. As shown in Fig. 3A and B, the mRNA and protein expression levels of NF- $\mathrm{NB}$ p65 and MCP-1 were significantly increased in the kidneys of diabetic rats compared to the normal control rats, and the increasing expression of NF- $\kappa$ B p65 correlated with a concomitant degradation of the inhibitory protein, I $\kappa \mathrm{B} \alpha$, in the cytosol. However, RLM treat-

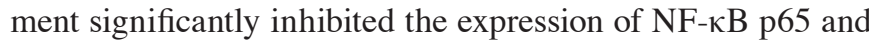
MCP-1 at both the mRNA and protein levels in the diabetic rats, and correlated with a concomitant increase in the expression of $\mathrm{I} \kappa \mathrm{B} \alpha$ protein. There were no significant differences in the expression of $\mathrm{NF}-\kappa \mathrm{B}$ p65 and MCP-1 between the normal control and control + RLM groups.

\section{Discussion}

$\mathrm{DN}$ is the major cause leading to end-stage renal disease. The pathological effects of DN include kidney hypertrophy, glomerular and tubular basement membrane thickening. This condition is further characterised by persistent proteinuria, hypertension and deterioration of renal function (24). Furthermore, mesangial matrix expansion and increased albuminuria closely correlate with the progression of renal failure (25). In the present study, STZ-induced diabetic rats showed significantly elevated blood glucose levels, higher KW/ BW ratios, as well as increased urine protein, urea nitrogen and Scr levels, which are indicative of kidney hypertrophy, glomerular injury and renal dysfunction (26). The mesangial matrix was more extensive in the glomeruli of diabetic rats than in normal control rats, and the GBM thickening and podocyte foot process effacement were striking in the diabetic rats. However, STZ-induced DM rats were treated with RLM for 24 weeks; we found that in the RLM-treated group, the diabetic parameters mentioned above were partially reverted, which suggests that RLM may effectively ameliorate renal injury in STZ-induced diabetic rats. Furthermore, there were no significant differences in the parameters mentioned above between the normal control and control + RLM groups, which therefore suggests that RLM treatment does not lead to renal injury in rats.

$\mathrm{OS}$ is defined as an excessive production of ROS surpassing the existing anti-oxidative defense mechanisms, and plays a critical role in the development and progression of diabetic complications, including DN (27). The markers of OS, such as ROS and reduced levels of anti-oxidants, have been found in the blood and renal tissues during both human and experimental diabetes (28). ROS play an important role in high 
glucose-induced renal injury. Numerous reports have shown that hyperglycemia generating $\mathrm{ROS}$, such as $\mathrm{O}_{2}^{-}$and $\mathrm{H}_{2} \mathrm{O}_{2}$, in renal mesangial or tubular cells contribute to the overproduction of extracellular matrix proteins and inflammation $(29,30)$. In addition, hyperglycemia attenuates anti-oxidative mechanisms through the glycation of scavenging enzymes, such as SOD and catalase. However, SOD is the first-line physiological defence against OS and, as an enzymatic anti-oxidant, SOD rapidly catalyses the dismutation of $\mathrm{O}_{2}{ }^{-}$and promotes its removal (31). The importance of SOD in the pathogenesis of diabetic renal lesions has been elegantly demonstrated by numerous studies (32). In DN animal models, the addition of the exogenous SOD mimetic tempol has shown encouraging results in the suppression of albuminuria $(33,34)$. In addition, tempol has been shown to diffuse through the cell membrane and react with both intracellular and extracellular ROS (34). Other studies have shown that urinary MDA levels are a reliable index for kidney damage (35). A previous study showed that type 2 diabetic patients with biopsy-diagnosed diabetic glomerular sclerosis have increased plasma and urinary MDA levels. Chang et al (36) demonstrated that increased urinary MDA levels are a consequence of increased glomerular MDA levels; they also found that both plasma and urinary levels of MDA, which is an important marker of lipid peroxidation, are markedly higher in patients with DN than in healthy controls. The urinary MDA levels significantly correlate with the degree of glomerulosclerosis and the index of mesangial expansion in DN patients (36). In the present study, kidney dysfunction resulting from diabetes was accompanied by a decrease in T-AOC and SOD levels, and increased levels of MDA and ROS in the kidneys, suggesting that heightened OS is involved in the impairment of renal function. The changes in T-AOC and SOD levels, MDA and ROS levels in rats with DN were reversed by RLM treatment, indicating that anti-OS could be one of the mechanisms by which RLM treatment alleviates kidney dysfunction in STZ-induced diabetic rats.

The kidney may be particularly vulnerable to early structural and functional damage caused by hyperglycemia-induced OS in diabetes (2). OS and inflammation induced by diabetes in the kidney are closely related events in the pathogenesis of DN. It is well known that the generation of reactive species by inflammatory cells induces OS. However, OS may induce inflammation through $\mathrm{NF}-\kappa \mathrm{B}$-mediated pro-inflammatory gene expression (37), and this intricate relationship has been demonstrated by numerous studies (38). Diabetes-induced glomerular macrophage infiltration and $\mathrm{NF}-\kappa \mathrm{B}$ activation are associated with albuminuria, mesangial expansion and glomerular hypertrophy in the kidney, which have been partially or completely prevented by anti-inflammatory agents, such as mycophenolate mofetil, erythromycin or methotrexate (39). In response to $\mathrm{OS}, \mathrm{NF}-\kappa \mathrm{B}$ is activated and allowed to translocate into the nucleus, where it enhances the transactivation of genes encoding cytokines, and leads to the increased production of many inflammatory cytokines, including MCP-1, that play an important role in the pathogenesis of DN (40). MCP-1 is a specific chemoattractant for monocytes and is implicated in the recruitment and activation of monocytes/macrophages to the glomerulus during proliferative glomerular disease; it is overexpressed in glomeruli, which precedes and closely correlates with macrophage infiltration (41). The importance of MCP-1 in early DN has been demonstrated using genetically deficient rats or the therapeutic blockade of the MCP-1 receptor, CCR2 (42,43). MCP-1 has also been recognised as a possible mediator of the development and progression of diabetic renal injury (44). However, numerous studies have shown that the activation of $\mathrm{NF}-\kappa \mathrm{B}$ plays an important role in MCP-1 expression in diabetic renal injury. In vitro and in vivo, high glucose levels or homocysteine stimulate MCP-1 mRNA and protein expression through the activation of NF- $\kappa \mathrm{B}(44,45)$. The stretching of mesangial cells also stimulates MCP-1 activity via an NF- $\mathrm{B}$-mediated MCP-1-dependent pathway, and this effect is prevented by rosiglitazone (41). In the present study, NF- $\kappa \mathrm{B}$ and MCP-1 mRNA and protein expression in the kidney were increased significantly in the diabetic rats, which also suggested the involvement of NF- $\mathrm{NB}$ and MCP-1 in the development of DN. Furthermore, RLM treatment significantly inhibited the mRNA and protein expression of $\mathrm{NF}-\kappa \mathrm{B}$ and MCP-1 in diabetic rats, and correlated with a concomitant increase in the expression of the $\mathrm{I} \kappa \mathrm{B} \alpha$ protein.

In conclusion, the present study demonstrates that systematic and local markers of OS are significantly increased in STZ-induced diabetic rats. Increased glomerular ROS levels are associated with decreased anti-oxidant levels and with a profound impairment in the oxidative-anti-oxidative balance in diabetic renal function. Furthermore, a NF- $\mathrm{B}-\mathrm{MCP}-1-$ dependent pathway may be involved in this impairment. RLM treatment was found to ameliorate these functional and histological abnormalities by improving glucose and lipid metabolism, reducing $\mathrm{OS}$ and inhibiting the expression of $\mathrm{NF}-\kappa \mathrm{B}$ and MCP-1. Further studies are required to understand the specific signaling pathway through which RLM exerts its protective effects on the diabetic kidney.

\section{Acknowledgements}

This study was supported by The Project Foundation of the Science and Technology Program of the Hunan Province (no. 2010FJ6025), the Hengyang Administration of Science and Technology (no. 2011KS1), and the Scientific Research Foundation from the Department of Ministry Health of the Hunan Province (no. B2007094).

\section{References}

1. Cooper ME: Is diabetic nephropathy disappearing from clinical practice? Pediatr Diabetes 7: 237-238, 2006.

2. Singh DK, Winocour P and Farrington K: Oxidative stress in early diabetic nephropathy: fueling the fire. Nat Rev Endocrinol 7: 176-184, 2011.

3. Kashihara N, Haruna Y, Kondeti VK and Kanwar YS: Oxidative stress in diabetic nephropathy. Curr Med Chem 17: 4256-4269, 2010.

4. Elmarakby AA and Sullivan JC: Relationship between oxidative stress and inflammatory cytokines in diabetic nephropathy. Cardiovasc Ther 30: 49-59, 2012.

5. Jones DP: Redefining oxidative stress. Antioxid Redox Signal 8: 1865-1879, 2006

6. Goth L: Reactive oxygen species, hydrogen peroxide, catalase and diabetes mellitus. Redox Rep 11: 281-282, 2006.

7. Fatehi-Hassanabad Z, Chan CB and Furman BL: Reactive oxygen species and endothelial function in diabetes. Eur $\mathrm{J}$ Pharmacol 636: 8-17, 2010.

8. Kaneto H, Katakami N, Matsuhisa M and Matsuoka TA: Role of reactive oxygen species in the progression of type 2 diabetes and atherosclerosis. Mediators Inflamm 2010: 453892, 2010 
9. Scandalios JG: Oxidative stress: molecular perception and transduction of signals triggering antioxidant gene defenses. Braz J Med Biol Res 38: 995-1014, 2005.

10. Liu WH, Hei ZQ, Nie H, et al: Berberine ameliorates renal injury in streptozotocin-induced diabetic rats by suppression of both oxidative stress and aldose reductase. Chin Med J 121: 706-712, 2008.

11. Firenzuoli F and Gori L: Herbal medicine today: clinical and research issues. Evid Based Complement Alternat Med 4 (Suppl 1): 37-40, 2007.

12. Rong J, Cheung CY, Lau AS, Shen J, Tam PK and Cheng YC: Induction of heme oxygenase-1 by traditional Chinese medicine formulation ISF-1 and its ingredients as a cytoprotective mechanism against oxidative stress. Int J Mol Med 21: 405-411, 2008.

13. Xu GJ, He HX, Xu LS and Jin RY: Chinese Materia Medical. Chinese Medicinal Science \& Technology Publishing House: Beijing 7: 223, 1998

14. Health. SPCotMoP: Pharmacopoeia of the People's Republic of China 2005. Chemical Industry Press, Beijing, P.R. China 1: 1, 2005.

15. Choi SJ, Kim MJ, Heo HJ, et al: Protective effect of Rosa laevigata against amyloid beta peptide-induced oxidative stress. Amyloid 13: 6-12, 2006.

16. Zhang QJ, Yin WD, Xi SM, et al: Effects of Fructus Rosa Laevigata and membrance of chickens gizzard on serum glucose, lipid and insulin levels in rabbits. Chin J Arterioscler 11: 227-229, 2003.

17. Zhou YJ, Liao QJ, Zhang QJ, Qin ZQ, Tang HL and Song Y: Experimental study of Rosa Laevigata Mickx in streptozotocininduced diabetic rats. J Nanhua Univ (Medical Edition) 35: 332-335, 2007.

18. Zhou YJ, Liao QJ, Zhang QJ and Qin Z: Effect of Rosa Laevigata on expression of MCP-1 in rats with diabetic nephropathy. Prog Mod Biomed 9: 4425-4427, 2009.

19. Levin-Iaina N, Iaina A and Raz I: The emerging role of NO and IGF-1 in early renal hypertrophy in STZ-induced diabetic rats. Diabetes Metab Res Rev 27: 235-243, 2011.

20. Ni T, Hu Y, Sun L, et al: Oral route of mini-proinsulin-expressing Ganoderma lucidum decreases blood glucose level in streptozocin-induced diabetic rats. Int J Mol Med 20: 45-51, 2007.

21. Bhaskaran N, Shukla S, Srivastava JK and Gupta S: Chamomile: an anti-inflammatory agent inhibits inducible nitric oxide synthase expression by blocking RelA/p65 activity. Int J Mol Med 26: 935-940, 2011

22. Livak KJ and Schmittgen TD: Analysis of relative gene expression data using real-time quantitative PCR and the 2(-Delta Delta C(T)). Methods 25: 402-408, 2001.

23. Jacob A, Rajan D, Pathickal B, et al: The inhibitory effect of ghrelin on sepsis-induced inflammation is mediated by the MAPK phosphatase-1. Int J Mol Med 25: 159-164, 2010.

24. Andersen AR, Christiansen JS, Andersen JK, Kreiner S and Deckert T: Diabetic nephropathy in Type 1 (insulin-dependent) diabetes: an epidemiological study. Diabetologia 25: 496-501, 1983.

25. Scivittaro V, Ganz MB and Weiss MF: AGEs induce oxidative stress and activate protein kinase C-beta(II) in neonatal mesangia cells. Am J Physiol Renal Physiol 278: F676-F683, 2000.

26. Danda RS, Habiba NM, Rincon-Choles H, et al: Kidney involvement in a nongenetic rat model of type 2 diabetes. Kidney Int 68 $2562-2571,2005$

27. Noh $\mathrm{H}$ and $\mathrm{Ha} \mathrm{H}$ : Reactive oxygen species and oxidative stress. Contrib Nephrol 170: 102-112, 2011
28. Giacco F and Brownlee M: Oxidative stress and diabetic complications. Circ Res 107: 1058-1070, 2010.

29. Ha $\mathrm{H}$ and Lee HB: Reactive oxygen species as glucose signaling molecules in mesangial cells cultured under high glucose. Kidney Int 77: S19-S25, 2000

30. Iglesias-De La Cruz MC, Ruiz-Torres P, Alcami J, et al: Hydrogen peroxide increases extracellular matrix mRNA through TGF-beta in human mesangial cells. Kidney Int 59: 87-95, 2001.

31. Fridovich I: Superoxide radical and superoxide dismutases. Annu Rev Biochem 64: 97-112, 1995.

32. DeRubertis FR, Craven PA, Melhem MF and Salah EM: Attenuation of renal injury in $\mathrm{db} / \mathrm{db}$ mice overexpressing superoxide dismutase: evidence for reduced superoxide-nitric oxide interaction. Diabetes 53: 762-768, 2004.

33. Peixoto EB, Pessoa BS, Biswas SK and Lopes de Faria JB Antioxidant SOD mimetic prevents NADPH oxidase-induced oxidative stress and renal damage in the early stage of experimental diabetes and hypertension. Am J Nephrol 29: 309-318, 2009.

34. Fujita H, Fujishima H, Chida S, et al: Reduction of renal superoxide dismutase in progressive diabetic nephropathy. J Am Soc Nephrol 20: 1303-1313, 2009.

35. Hermanns RC, de Zwart LL, Salemink PJ, Commandeur JN, Vermeulen NP and Meerman JH: Urinary excretion of biomarkers of oxidative kidney damage induced by ferric nitrilotriacetate. Toxicol Sci 43: 241-249, 1998.

36. Chang JM, Kuo MC, Kuo HT, Chiu YW and Chen HC: Increased glomerular and extracellular malondialdehyde levels in patients and rats with diabetic nephropathy. J Lab Clin Med 146: 210-215, 2005.

37. Calcutt NA, Cooper ME, Kern TS and Schmidt AM: Therapies for hyperglycaemia-induced diabetic complications: from animal models to clinical trials. Nat Rev Drug Discov 8: 417-429, 2009.

38. Kern TS: Contributions of inflammatory processes to the development of the early stages of diabetic retinopathy. Exp Diabetes Res 2007: 95103, 2007.

39. Yozai K, Shikata K, Sasaki M, et al: Methotrexate prevents renal injury in experimental diabetic rats via anti-inflammatory actions. J Am Soc Nephrol 16: 3326-3338, 2005.

40. Tesch GH: MCP-1/CCL2: a new diagnostic marker and therapeutic target for progressive renal injury in diabetic nephropathy. Am J Physiol Renal Physiol 294: F697-F701, 2008.

41. Gruden G, Setti G, Hayward A, et al: Mechanical stretch induces monocyte chemoattractant activity via an NF-kappaB-dependent monocyte chemoattractant protein-1-mediated pathway in human mesangial cells: inhibition by rosiglitazone. J Am Soc Nephrol 16: 688-696, 2005.

42. Chow FY, Nikolic-Paterson DJ, Ma FY, Ozols E, Rollins BJ and Tesch GH: Monocyte chemoattractant protein-1-induced tissue inflammation is critical for the development of renal injury but not type 2 diabetes in obese $\mathrm{db} / \mathrm{db}$ mice. Diabetologia 50 : 471-480, 2007.

43. Kanamori H, Matsubara T, Mima A, et al: Inhibition of MCP-1/ CCR2 pathway ameliorates the development of diabetic nephropathy. Biochem Biophys Res Commun 360: 772-777, 2007.

44. Ha H, Yu MR, Choi YJ, Kitamura M and Lee HB: Role of high glucose-induced nuclear factor-kappaB activation in monocyte chemoattractant protein-1 expression by mesangial cells. J Am Soc Nephrol 13: 894-902, 2002.

45. Hwang SY, Woo CW, Au-Yeung KK, Siow YL, Zhu TY and O K Homocysteine stimulates monocyte chemoattractant protein-1 expression in the kidney via nuclear factor-kappaB activation. Am J Physiol Renal Physiol 294: F236-F244, 2008. 